\title{
Interactive Super-resolution through Neighbor Embedding
}

\author{
Jian $\mathrm{Pu}^{1}$, Junping Zhang ${ }^{1 \star}$, Peihong Guo ${ }^{2}$, and Xiaoru Yuan ${ }^{2}$ \\ 1 Shanghai Key Lab of Intelligent Information Processing \\ School of Computer Science, Fudan University, Shanghai 200433, China \\ ${ }^{2}$ Key Laboratory of Machine Perception (Ministry of Education) \\ School of EECS, Peking University, Beijing 100871, China
}

\begin{abstract}
Learning based super-resolution can recover high resolution image with high quality. However, building an interactive learning based super-resolution system for general images is extremely challenging. In this paper, we proposed a novel GPU-based Interactive Super-Resolution system through Neighbor Embedding (ISRNE). Random projection tree (RPtree) with manifold sampling is employed to reduce the number of redundant image patches and balance the node size of the tree. Significant performance improvement is achieved through the incorporation of a refined GPU-based brute force $\mathrm{kNN}$ search with a matrix-multiplicationlike technique. We demonstrate 200-300 times speedup of our proposed ISRNE system with experiments in both small size and large size images.
\end{abstract}

\section{Introduction}

Learning based super-resolution, which recovers the high resolution (HR) by learning the relationship between HR training images and low resolution (LR) counterparts, attracts much attention recently. Comparing with other methods, learning based super-resolution is capable of extracting more image/patch information from a collection of image pairs or image patch pairs and supports higher magnification factors with fewer LR images [1].

Freeman et al. [2] constructed a Markov network to connect the LR and HR image patches, followed by employing Bayesian belief propagation to allocate HR counterparts. However, artistic style is exhibited in the recovered HR image [3]. Assuming local geometry preservation for both high- and low-dimensional manifold, Chang et al. [4] proposed single image super-resolution through neighbor embedding (SRNE). Wei et al. [5] improved SRNE by extracting visual primitives and removing the noisy patches by validation (NEVPM). Yang et al. [6] investigated the selection of neighbor factors from the perspective of compressed sensing and Su et al. [7] studied the influence of patches from the viewpoint of neighborhood preservation. Furthermore, efficient face super-resolution methods has been proposed by employing a hallucination algorithm and its variants [8-10]. These

\footnotetext{
* Corresponding author.
} 
algorithms are efficient because they employ linear PCA algorithm to project all the LR images into a low-dimensional subspace [8].

It is worth noting that although many refinements have been proposed, the performance issue of learning based super-resolution, which plays a crucial role in its practical applications, is not well studied yet. It is very challenging to build an interactive (a few frames per second) learning-based method which can learn from hundreds of thousands of image patches or training images.

To attack the aforementioned issue, we propose Random Projection tree (RPtree) [11] with manifold sampling to reduce the total number of image patches and balance the tree structure. Then a refined GPU-based kNN search is employed to find the neighboring patches from the nodes of RPtree. Thirdly, we perform SRNE for the recovery of high-resolution test image patches. Finally, we introduce a global reconstruction constrain for further improving the image quality of high-resolution images. We successfully build an interactive superresolution with neighboring embedding system (ISRNE) without compromising the image quality, even achieving a slightly better quality than the previous SRNE. Our experiments indicate that ISRNE not only demonstrates a remarkable improvement over performance, but also shows a superior scalability to existing methods.

The rest of the paper is organized as follows. In Sect. 2, we describe our interactive SRNE in details. We report the experiment results and discuss the limitation of the ISRNE in Sect. 3, followed by a conclusion in Sect. 4.

\section{Interactive Super-resolution through Neighbor Embedding (ISRNE)}

To construct an interactive super-resolution system, we devote our efforts on the parallelization of two crucial steps of SRNE. These steps are: (1) All the image patches are separately reconstructed by neighbor embedding which has a closedform solution; (2) The neighboring patches of each test patch are searched by kNN. It is worth noting that in this paper, we do not consider the parallelization of NEVPM, a refined version of SRNE since NEVPM comprises some steps which are hard to be paralleled [5]. For self-containness, we give a brief introduction on SRNE before the discussion of our approach.

\subsection{Super-resolution through Neighbor Embedding}

A key assumption in SRNE is that the local geometries of image patches are similar in two distinct HR and LR feature spaces. Therefore, an HR counterpart of an LR image patch can be recovered using a collection of HR training patches.

We denote $\mathbf{y}_{s}$ and $\mathbf{x}_{s}$ be the training patches from HR images and its LR counterparts respectively. The optimal reconstruction weights of each patch $\mathbf{x}_{t}$ from LR test images is obtained by minimizing the local reconstruction error [4]:

$$
\epsilon=\min \left\|\mathbf{x}_{t}-\sum_{\mathbf{x}_{s} \in \mathcal{N}_{\mathbf{x}_{t}}} \omega_{\mathbf{t s}} \mathbf{x}_{s}\right\|^{2}, \quad \mathbf{x}_{t}, \mathbf{x}_{s} \in \mathbb{R}^{d}
$$


subject to the constraints $\sum_{\mathbf{x}_{s} \in \mathcal{N}_{\mathbf{x}_{t}}} \omega_{\mathbf{t s}}=1$ and $\omega_{\mathbf{t s}}=0$ for any $\mathbf{x}_{s} \notin \mathcal{N}_{\mathbf{x}_{t}}$, where $\mathcal{N}_{\mathbf{x}_{t}}$ is the neighborhood of $\mathbf{x}_{t}$ given neighbor factor $k$. Denote $\mathbf{G}=\left(\mathbf{x}_{t} \mathbf{1}^{T}\right.$ $\left.\mathbf{X}_{s}\right)^{T}\left(\mathbf{x}_{t} \mathbf{1}^{T}-\mathbf{X}_{s}\right)$, Equation (1) can be optimized by solving a linear system equation $\mathbf{G W}=1$, s.t. $\sum_{\mathbf{x}_{s}} \mathbf{w}_{t s}=1$. Thus, the HR patch $\mathbf{y}_{t}$ is attained as follows:

$$
\mathbf{y}_{t}=\sum_{\mathbf{x}_{s} \in \mathcal{N}_{\mathbf{x}_{t}}} \omega_{\mathrm{ts}} \mathbf{y}_{s}, \quad \mathbf{y}_{s} \in \mathbb{R}^{D} .
$$

where $\mathbf{y}_{s}$ is the HR counterpart of LR image patch $\mathbf{x}_{s}$.

In the last step above, we can either simply average the values in the overlapped regions between adjacent patches, or employ other more sophisticated methods (e.g. one pass algorithm) to enforce inter-patch relationship.

To achieve efficient parallelization of SRNE, we introduce RPtree with manifold sampling.

\subsection{Random projection tree with manifold sampling}

RPtree, a variant of the $k$-d tree, hierarchically partitions data of $D$ dimension into pieces in a manner which only depends on the intrinsic low-dimensional manifold [11]. The advantages of utilizing RPtree are that: (1) RPtree consumes less time $(O(n))$ to construct than PCA tree $(O(n \log n))$ [11], given $n$ data points; (2) Unlike $k$-d tree, RPtree can deal with high-dimensional data $(\geq 30)$ which are often seen in super-resolution domain; (3) RPtree won't bring a detrimental effect on the SRNE performance, since the manifold assumption of RPtree is the same as that of neighbor embedding [4].

RPtree is built in a streaming manner [12] in which the following statistics at each internal node of the tree are maintained and updated as new data arrives.

$$
\begin{gathered}
\mu_{i} \leftarrow\left(1-\alpha_{i}\right) \mu_{i-1}+\alpha_{i} x_{i} \\
\sigma_{i}^{2} \leftarrow\left(1-\alpha_{i}\right) \sigma_{i-1}^{2}+\alpha_{i}\left(x_{i}-\mu_{i}\right)^{2} .
\end{gathered}
$$

where $\mu_{i}$ and $\sigma_{i}^{2}$ denote the mean value and the corresponding variance in the $i$ th iterative times, respectively. Parameter $\alpha_{i}(\leq 1 / i)$ is a weighted factor, and $x_{i}$ means a new sample. The details can be seen in [12].

With this way, Freund et al. [11] proved that if data in $C$ are of intrinsic dimension $d$, the average diameter of any descendant cell ( $\leq d$ levels below) of $C$ will be less than half of the average diameter of $C$ with constant probability, picking any cell $C$ in the RP tree. However, this theorem is not sufficient to assure the even partition of RPtree especially when the data is distributed unevenly, which is the case in natural image patches. Since the background patches are quite similar, most of such patches will be assigned to few nodes. To reduce the difference of distribution density between the background and foreground patches, we introduce manifold sampling in order to balance the RPtree. Given $d$ dimensional $n$ data points $\mathbf{x}_{i}(i=1, \ldots, n)$, specifically, we approximate the geodesic distance between data points $\mathbf{x}_{i}$ and $\mathbf{x}_{j}$, which are lying close together (their Euclidean distances $\operatorname{dist}\left(\mathbf{x}_{i}, \mathbf{x}_{j}\right)$ is less than a given threshold $\epsilon$ or one 
of the $K$ nearest neighbors of $\mathbf{x}_{j}$ of $\mathbf{x}_{j}$ ), can be approximated. For data points lying far apart, we then approximate the geodesic distance by the shortest path distance which is formulated as follows:

$$
\operatorname{dist}\left(\mathbf{x}_{i}, \mathbf{x}_{j}\right)=\min \left\{\operatorname{dist}\left(\mathbf{x}_{i}, \mathbf{x}_{j}\right), \operatorname{dist}\left(\mathbf{x}_{i}, \mathbf{x}_{k}\right)+\operatorname{dist}\left(\mathbf{x}_{k}, \mathbf{x}_{j}\right)\right\} \quad \forall k, 0 \leq k \leq n
$$

Once the approximated geodesic distances between sample pairs in the high dimensional space are calculated, we can directly use these distance values to decimate redundant points in the manifold. We also prove an error upper bound of manifold sampling for super-resolution as follows:

$$
\frac{\|\Delta \mathbf{y}\|}{\|\mathbf{y}\|} \leq \frac{4 \epsilon}{\|\mathbf{P}\|}+\frac{r \epsilon}{\|\mathbf{y}\|},
$$

where $\mathbf{P}^{T} \mathbf{P}=\mathbf{G}$, and $r$ denotes the super-resolution factor. It indicates that we can guarantee the difference between the original SR results and correspond one with manifold sampling is small by carefully choosing $\epsilon$. Due to limited space, we put the proof into the supplementary.

\subsection{GPU Acceleration}

The complexity of the brute force kNN algorithm reaches an order of $O(n m d+$ $n m \log m$ ), where $m$ represents for the number of query data points and, $n$ for the number of reference data points, and $d$ is the dimensionality of the data points. To reduce computational cost, Garcia et al. [13] proposed a fast kNN search scheme based on a brute force GPU algorithm and obtained a remarkable increase in performance. However, a naïve implementation of GPU acceleration has very low efficiency due to large amount of un-coalesced memory access and bank conflicts. In our approach, we develop a matrix-multiplication-like algorithm, to compute the distances.

Denoting the query points as $\mathbf{X}_{t}\left(x_{t_{i j}}\right)_{m \times d}$ and the reference points as $\mathbf{X}_{s}\left(x_{s_{i j}}\right)_{n \times d}$, the squared distance matrix $\mathbf{D}\left(d i s t_{i j}^{2}\right)_{m \times n}$ can then be calculated:

$$
\mathbf{D}=\mathbf{X}_{t} \odot \mathbf{X}_{s}^{T}
$$

where $\odot$ denotes the operator between two matrices, and is defined by:

$$
d i s t_{i j}^{2}=\mathbf{x}_{t_{i}} \cdot \mathbf{x}_{s_{j}}=\sum_{k=1}^{d}\left(\mathbf{x}_{t_{i_{k}}}-\mathbf{x}_{s_{j_{k}}}\right)^{2} .
$$

Above approach is very similar to the matrix multiplication between $\mathbf{X}_{t}$ and $\mathbf{X}_{s}^{T}$, except that the computing kernel is modified. We can very conveniently cope with the computation utilizing currently available parallel matrix multiplication algorithms to obtain significant performance improvement. An illustration of this operation is shown in Fig. 1.

As the neighborhood number $k$ is relatively small in our application, we employ insertion sort for best performance [13]. Unlike Bishop et al.'s method [14], 


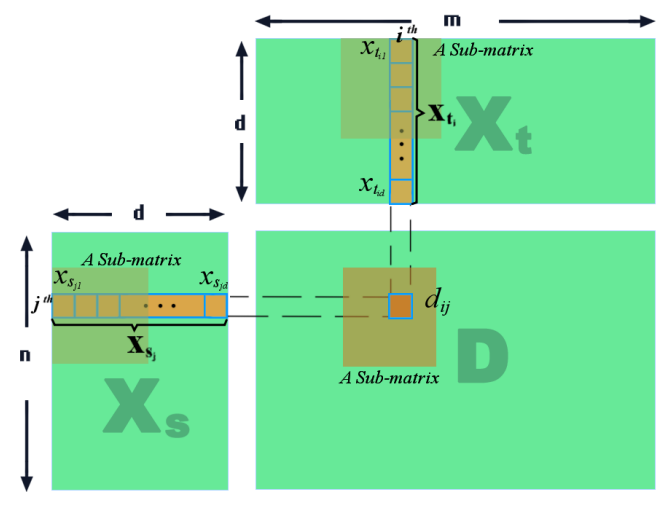

Fig. 1. Matrix-mulitplication-like operation between $\mathbf{X}_{\mathbf{t}}\left(x_{t_{i j}}\right)_{m \times d}$ and $\mathbf{X}_{\mathbf{s}}\left(x_{s_{i j}}\right)_{n \times d}$. We follow the method of matrix multiplication to compute the distance matrix, with the computing "kernel" modified.

we needn't perform dimension reduction which is required for $k$ nearest neighbor search on $k$-d tree. We further propose a combination of GPU-based kNN and RPtree with manifold sampling to speed up queries. In Sect. 3, we show that there is no negative impact on the the quality of restoration.

\subsection{Global Reconstruction Constrain}

In super-resolution domain, there are two important criteria to evaluate the recovered HR quality. One is the recovery of high frequency, the other is the consistency of LR and HR images. To keep the consistency, generally speaking, a global reconstruction constrain which means the simulated downsampling of the reconstructed HR image $\mathbf{Y}$ is the same as the LR image $\mathbf{X}$ should be considered as follows:

$$
\mathbf{X}=D_{S} H \mathbf{Y}
$$

where $H$ represents a blurring filter, and $D_{S}$ the downsampling operator.

As the consistency of LR and HR images are not always satisfied when patch by patch restoration is employed, Liu et al. [15] first proposed a two step method to combine the local nonparametric and global parametric model. In ISRNE, we utilize the back-projection method (BP) which has been studied by Yang et al. [6] as a post-processing procedure to enhance the consistency.

Back-projection algorithm is an iterative procedure for estimating unknown scenes. Given an estimation of the super resolution image, a simulated imaging process yields a set of low-resolution images which are compared with the observed low resolution images. The super-resolution estimation is then modified so as to reduce the error between the observed and simulated images. The process is terminated when the error is less than a predetermined level, or after a given 
number of iterations. The iterative procedure is formally described as follows:

$$
\begin{aligned}
\hat{\mathbf{Y}}^{(t+1)} & =\hat{\mathbf{Y}}^{(t)}+\mathbf{H}^{B P}\left(\mathbf{X}-\hat{\mathbf{X}}^{(t)}\right) \\
& =\hat{\mathbf{Y}}^{(t)}+\mathbf{H}^{B P}\left(\mathbf{X}-D_{S} H \hat{\mathbf{Y}}^{(t)}\right)
\end{aligned}
$$

where $\mathbf{H}^{B P}$ is a back-projection operator which is an approximation to the inverse of the operator $D_{S} H, t$ is the iterative times.

\section{Experiments}

In order to evaluate the effectiveness of ISRNE, experiments are carried out on two image sets of different scales. The smaller training set is from Chang et al.'s paper [4] as in Fig. 2, and the larger one consists of 370 images are collected from a well-known COREL database.

In the experiments, we will magnify the input image by a factor of 4 . In the LR images, we always use $3 \times 3$ LR patches, with overlap of 2 pixel between adjacent patches, corresponding to $12 \times 12$ patches with overlap of 8 pixels for the HR images. Like SRNE [4], the neighbor factor $k$ is set to be 5 and the features (first-order and second-order gradient) are extracted directly from the illuminance component of LR images since humans are more sensitive to illuminance changes.

The computer used for the evaluation is an Intel Core 2 Duo E7200 $2.53 \mathrm{GHz}$ with $2 \mathrm{~GB}$ of DDR2 memory PC2-5300 $(2 \times 1 \mathrm{~GB}$ dual-channel memory). The graphics card used is an NVIDIA GeForce 8800 GT with 512MB of DDR3 memory. NVIDIA CUDA 2.0 is employed for GPU computation.

We first evaluate the effectiveness of RPtree with manifold sampling using the small training set. The tree height is set to be 4, which is a tradeoff between the tree search and node search cost. To remove the randomness of RPtree, the output, i.e. the number of patches in each node, of 10 times are recorded. The sum of node frequencies of the 10 repetitions is reported as in Fig. 3. It is easy to see from Fig. 3 that the distribution of these patches on the tree nodes are highly imbalanced. Furthermore, there always exists a "big" node in the tree, which contains almost half of the total patches. For example, the frequency of a node owing the number of points in $[4000,6000]$ is equal to 10 . It is not difficult to observe that such patches are sampled from the background. After manifold sampling is employed, the imbalance is greatly reduced as shown in Fig. 3. It indicates that the proposed RPtree with manifold sampling indeed remove redundant patches and improve the balance of RPtree.

As shown in Fig. 4, we compare our method with neighbor embedding (SRNE) [4] and back projection. It is easy to notice that the forehead hair and double eye of the girl utilizing ISRNE are more clear than those using SRNE. Furthermore, ISRNE has lower RMSE for the recovery of HR image than SRNE, and is comparable to SRNE+BP method and sparse representation method [6] which needs several hours to get the result. It indicates that while the main goal of the proposed 

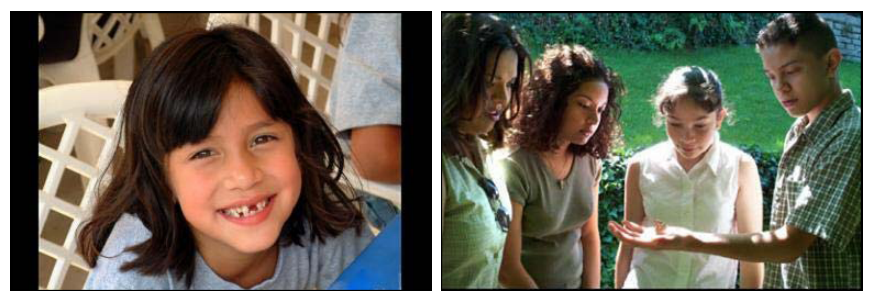

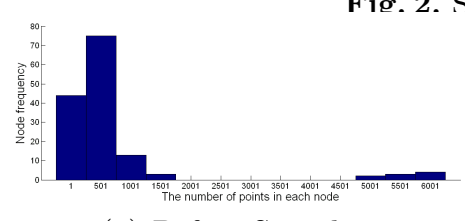

(a) Before Sampling

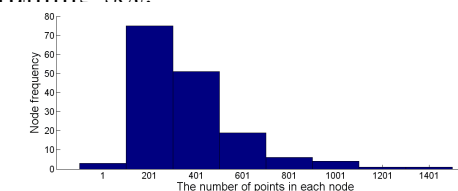

(b) After Sampling

Fig. 3. The distribution of data in RPtree. The vertical axis means the times of a node of RPtree owing the number of points in some interval.

ISRNE is to attain interactivity, the recovered image quality is not sacrificed, even better than that of the previous one (e.g. SRNE) in some cases.
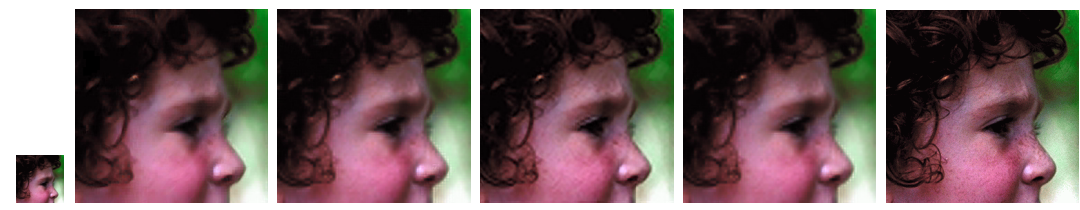

Fig. 4. $4 \times$ recovery of low resolution image. The root mean squared errors (RMSEs) are shown in the Brackets, from left to right: LR, SRNE (0.0387), SRNE + BP (0.0368), Sparse Representation (0.0357), ISRNE (0.0369), Ground truth.

To evaluate the performance of ISRNE, we compare it with several state-ofthe-art acceleration methods including: Brute force kNN search (BF), RPtree, partial distance search acceleration (PDS) [16], and the combination of manifold sampling with the former three methods. Furthermore, we also study the performance of three variants of the proposed ISRNE algorithm by neglecting either manifold sampling or RPtree, or both. The training images are the small training set as in Fig. 2. The test images of increasing sizes include one image from Chang et al.'s paper [4] and three images downloaded from Internet. To remove the randomicity of RPtree, all the methods related to RPtree are repeated 10 times and the corresponding means and standard deviations of the computational times are reported in Fig. 5.

It can be seen from Fig. 5 that compared with other methods, ISRNE has the best performance, and the acceleration ratio of $\mathrm{BF}$ is increased with the resolution of the test images. Furthermore, RPtree and manifold learning are two comparable and complementary methods which can further accelerate the refined GPU-based kNN procedure. When the size of images are smaller than $500 \times 348$, the running time is less than 1 second which is a basic requirement to 

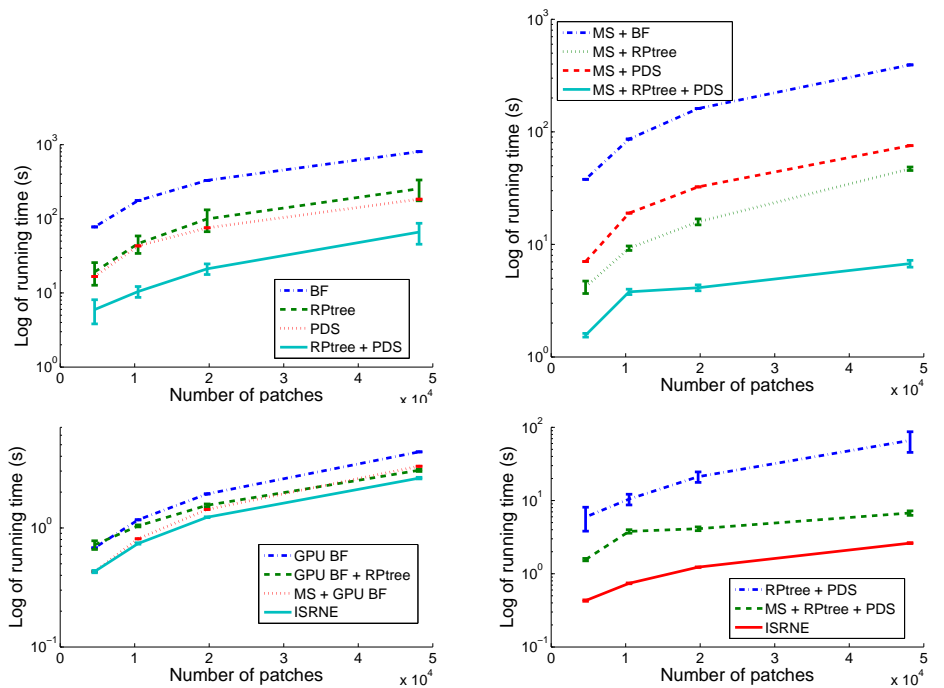

Fig. 5. A performance comparison on the super-resolution with different search methods for images with different resolutions.
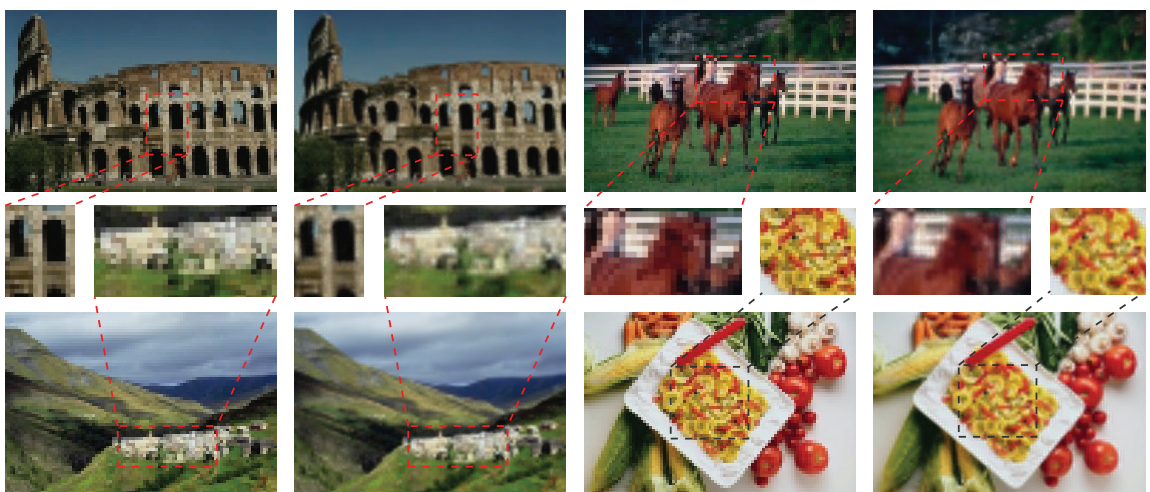

Fig. 6. $4 \times$ recovery of low resolution image using the big training set. For each scene, the left one is the LR image and the right one is recovered by ISRNE. From left to right, top and bottom, the RMSE errors of these four test images are: 0.0827, 0.0583, 0.0643, and 0.0734 , respectively. In the middle, there are close-ups of the four images. 
build an interactive super-resolution system. While the "MS+RPtree+PDS" made a remarkable improvement in the aspect of acceleration, it is hard for PDS to design an alternatively parallelized one due to its serial computation nature.

\begin{tabular}{|l|c|c|c|c|}
\hline & Test 1 & Test 2 & Test 3 & Test 4 \\
\hline$T_{\text {pre }}(\mathrm{s})$ & 9.490 & 9.450 & 9.420 & 9.400 \\
$T_{k N N}(\mathrm{~s})$ & 1.930 & 1.890 & 1.760 & 1.870 \\
$T_{w}(\mathrm{~s})$ & 0.115 & 0.118 & 0.117 & 0.118 \\
$T_{\text {resto }}(\mathrm{s})$ & 0.161 & 0.160 & 0.162 & 0.166 \\
$T_{\text {post }}(\mathrm{s})$ & 0.107 & 0.107 & 0.103 & 0.107 \\
\hline Total & 11.803 & 11.725 & 11.500 & 11.560 \\
\hline
\end{tabular}

Table 1. Running time (RT) of ISRNE in seconds, from top to bottom: RT for preprocessing, RT for kNN search, RT for weights calculation, RT for reconstruction, RT for postprocessing and the total RT.

Finally, we attempt to figure out how many training/test patches will hinder the interactive property of ISRNE. Therefore, we extract 131, 523 patches from $2,156,360(5828 \times 370)$ raw patches which are generated from the mentioned large training image set. The test set consists of four images from Corel test set without overlapping with the large training set. The HR test images (of size $384 \times 256$ ) recovered by ISRNE are shown in Figures 6 , and running time analysis are tabulated in Table 1. Compared with the LR images, we can observe that (1) the wall of Roman relics becomes cleaner; (2) the faces of these horses become smoother and a little bit recognizable; (3) some buildings on the mountain are shown up; (4) the food details in the dish is distinguishable and the edges of the dish is cleaner. Note that most of the running time is spent on pre-processing, especially on transferring all the 131,523 patches into memory. The time for kNN search and neighbor embedding is only about 2 second, while other aforementioned learning methods for super-resolution never run through such tough tests.

\section{Conclusion}

With the combination of RPtree with manifold learning and the refined GPUbased kNN search, an interactive super-resolution through neighbor embedding is proposed. Experiments on both small-scale images and large-scale images indicate that the proposed ISRNE not only remarkably accelerates the procedure of super-resolution but also enhances the image quality of recovered high-resolution images well. Note that although the kNN search time grows with the training patches and test patches, a more powerful hardware will greatly neutralize the side effect. Meanwhile, this scheme can easily be applied to other patch based search and replace problems like image denoising, inpainting, texture transfer, and transparency separation.

There are a few issues that require a thorough investigation. Since RPtree can construct a tree much more efficient than other tree-based algorithms, we 
will investigate how to dynamically update the training patches to further refine the performance of ISRNE in the future. Furthermore, we would like to generalize the proposed framework to other learning based super-resolution method.

\section{Acknowledgements}

This research was sponsored by the 973 Program (No. 2010CB327900 and No. 2009CB320903), 863 Project (2007AA01Z176), NSFC 60635030 and Key Project of Chinese Ministry of Education (No. 109001).

\section{References}

1. Lin, Z.C., He, J.F., Tang, X.O., Tang, C.K.: Limits of learning-based superresolution algorithm. In: Proceedings of IEEE CVPR. (2007) 1-7

2. Freeman, W.T., Pasztor, E.C., Carmichael, O.T.: Learning low-level vision. International Journal of Computer Vision 40(1) (2000) 25-47

3. Freeman, W.T., Jones, T.R., Pasztor, E.C.: Example based superresolution. IEEE Computer Graphics and Applications 22(2) (2002) 56-65

4. Chang, H., Yeung, D.Y., Xiong, Y.: Super-resolution through neighbor embedding. In: Proceedings of IEEE CVPR. (2004) 275-282

5. Wei, F., Yeung, D.Y.: Image hallucination using neighbor embedding over visual primitive manifolds. In: Proceedings of IEEE CVPR. (2007) 1-7

6. Yang, J.C., Wright, J., Ma, Y., Huang, T.: Image super-resolution as sparse representation of raw image patches. In: Proceedings of IEEE CVPR. (2008) 1-8

7. Su, K., Tian, Q., Xue, Q., Sebe, N., Ma, J.: Neighborhood issue in single-frame image super-resolution. In: Proceedings of IEEE ICME. (2005) 1122-1125

8. Gunturk, B.K., Batur, A.U., Altunbasak, Y., Hayes III, M.H., Mersereau, R.M.: Eigenface-domain super-resolution for face recognition. IEEE Trans. Image Proc. 12(5) (2003) 597-606

9. Liu, C., Shum, H.Y., Freeman, W.T.: Face hallucination: Theory and practice. International Journal of Computer Vision 75(1) (2007) 115-134

10. Baker, S., Kanade, T.: Limits on super-resolution and how to break them. IEEE Trans. PAMI 24(9) (2002) 1167-1183

11. Freund, Y., Dasgupta, S., Kabra, M., Verma, N.: Learning the structure of manifolds using random projections. In: NIPS. (2008) 473-480

12. Dasgupta, S., Freund, Y.: Random projection trees and low dimensional manifolds. Technical Report CS2007-0890, UCSD (2007)

13. Garcia, V., Debreuve, E., Barlaud, M.: Fast k nearest neighbor search using gpu. In: CVPR Workshop on Computer Vision on GPU. (2008) 1-7

14. Bishop, C.M., Blake, A., Marthi, B.: Super-resolution enhancement of video. In: Proceedings 9th International Conference on Artificial Intelligence and Statistics. (2003)

15. Liu, C., Shum, H.Y., Zhang, C.S.: A two-step approach to hallucinating faces: Global parametric model and local nonparametric model. In: Proceedings of IEEE CVPR. (2001) 192-198

16. Qiao, Y.L., Pan, J.S., Sun, S.H.: Improved partial distance search for k nearestneighbor classification. In: Proceedings of IEEE ICME. Volume 2. (2004) 12751278 